\title{
Stoichiometry of Receptors at the Plasma Membrane During Their Endocytosis Using Total Internal Reflection Fluorescent (TIRF) Microscopy Live Imaging and Single-Molecule Tracking
}

\section{Laura Salavessa and Nathalie Sauvonnet}

\begin{abstract}
Determination of protein stoichiometry in living cells is key to understanding basic biological processes. This is particularly important for receptor-mediated endocytosis, a highly regulated mechanism that requires the sequential assembly of numerous factors. Here, we describe a quantitative approach to analyze receptor clustering dynamics at the plasma membrane. Our workflow combines TIRF live imaging of a CRISPR-Cas9-edited cell line expressing a GFP-tagged receptor in a physiological relevant environment, a calibration technique for single-molecule analysis of GFP, and detection and tracking with an open-source software. This method allows to determine the number of receptor molecules at the plasma membrane in real time.
\end{abstract}

Key words Single-molecule tracking (SPT), TIRF, Endocytosis, Photobleaching, Receptor, Dynamics, Plasma membrane, Quantitative biology

\section{Introduction}

Many proteins are part of multimeric complexes, often containing different number and type of subunits, and oligomerize as part of their catalytic activity or function. Such proteins play a critical role in a wide variety of cellular events, which is particularly notable in membrane proteins that drive ion and protein transport and homeostasis, signal transduction, endocytosis, and secretion. Therefore, studying their stoichiometry and dynamics, under native conditions in living cells, is key to better understand their function and the cellular mechanisms in which they participate.

In this introduction, we will briefly present some examples reported in the literature, describing the link between protein stoichiometry and function.

Florence Niedergang, Nicolas Vitale and Stéphane Gasman (eds.), Exocytosis and Endocytosis: Methods and Protocols, Methods in Molecular Biology, vol. 2233, https://doi.org/10.1007/978-1-0716-1044-2_1,

(C) Springer Science+Business Media, LLC, part of Springer Nature 2021 
One of the first well-studied examples is the Epidermal Growth Factor Receptor (EGFR). Although largely accepted that ligandinduced dimerization of EGFR is essential for signal transduction, some studies showed that EGFR activation is also associated with the formation of high-order oligomers that efficiently propagate the signal [1]. Indeed, several studies analyzing EGFR stoichiometry using Förster Resonance Energy Transfer (FRET), Fluorophore Localization Imaging with Photobleaching (FLImP), or biochemical techniques like cross-linking experiments [2] revealed that formation of at least receptor tetramers was necessary for higher tyrosine kinase activity [1,3]. Moreover, mutations blocking EGFR multimerization have been shown to reduce its autophosphorylation and the activation of downstream signaling partners $[3,4]$. Altogether, these findings show that EGF signaling is regulated by receptor clustering, which is of particular interest since mutations in EGFR that lead to enhanced signaling are frequently associated with cancer.

Another example for which receptor stoichiometry has been linked to its function is CC-chemokine receptor 5 (CCR5), a G Protein-Coupled Receptor (GPCR) and the main coreceptor for human immunodeficiency virus type 1 (HIV-1). Different affinities of the virus for CCR5 have been proposed to regulate HIV virulence, resistance to inhibitors, and cellular tropisms. In particular, using bimolecular fluorescence complementation (BiFC) and FRET assays, it was shown that various CCR5 populations are present at the cell surface and that these exist in a variety of conformations due to different dimerization interfaces. Furthermore, it was shown that HIV-1 preferentially recognizes CCR5 monomers as opposed to dimers $[5,6]$, in agreement with findings that anti-CCR5 monoclonal antibodies that induce receptor dimerization prevent HIV-1 infection [7]. Therefore, the oligomerization state of CCR5 seems to regulate HIV interaction, uptake, and infection.

It should be noted that, for most of the stoichiometry analysis summarized above, EGFR and CCR 5 were ectopically expressed in cells, which might result in a biased analysis due to the nonphysiological expression of the receptors.

Another mechanism for which protein stoichiometry has been shown to be determinant is endocytosis. The highly characterized clathrin-mediated uptake is a process involving many factors [8], although the orchestration of these factors and their organization is not fully understood. Recent advances in gene-editing tools and single-molecule fluorescence imaging techniques enabled the characterization of protein oligomerization and complex assembly at the plasma membrane in living cells. For instance, in clathrinmediated endocytosis, pits initiate by coordinated arrival of one clathrin triskelion and two membrane-bound AP2 proteins that stabilize the complex, increasing its residence time at the membrane 
and allowing further addition of more clathrin molecules [9]. In mammals, the last step of vesicle fission requires the action of the large GTPase dynamin. Recently, several groups showed that approximately 26 molecules of dynamin are recruited to the neck of the clathrin vesicle $[10,11]$, which is sufficient to form a short helix that encircles the neck of the vesicle once and bridges two dynamin molecules together, thus activating the GTPase and driving scission [10].

Altogether, these studies demonstrate that understanding protein stoichiometry and assembly is necessary to provide new insights, not only in its physiological function but also in its biogenesis, its targeting by pathogens, and its deregulation in disease.

In this chapter, we will describe our method to infer in realtime the number of proteins at the plasma membrane, using an endogenously expressed gene-edited receptor coupled to a fluorescent tag and single-molecule analysis. To quantify the number of molecules at the plasma membrane, we take advantage of the high signal-to-noise ratio provided by TIRF microscopy. Our receptor model is the $\gamma$ chain of the interleukin-2 receptor (IL-2R $\gamma$ ), a critical signaling receptor for immune function and a fine example of a clathrin- and caveolin-independent endocytic cargo $[12,13]$. The receptor was found to accumulate at the base of membrane protrusions, suggesting this could constitute the initial step of endocytic pit formation [14]. This endocytic process does not depend on any coat protein, thus raising the question as to whether local receptor clustering may initiate membrane bending. To test this hypothesis, we developed a workflow to analyze IL-2R $\gamma$ stoichiometry during endocytosis in living $\mathrm{T}$ lymphocytes.

Our protocol combines TIRF live imaging of CRISPR-Cas9edited cells that endogenously express the receptor of interest coupled to eGFP, with a calibration method for single-molecule analysis using purified eGFP to determine the unitary bleaching step of the fluorophore. The intensity of a single eGFP molecule corresponds to the loss of fluorescence signal observed in one photobleaching step. This intensity standard (unitary bleaching step) is then used to infer the number of receptor molecules, in live-cell TIRF images, that are tracked with a plug-in implemented in the open-source software Icy (Fig. 1). Altogether, this method reports the number of receptor molecules at the plasma membrane during endocytosis, in real time.

\section{Materials}

\subsection{Coverslips and Cell Preparation}

1. CRISPR-Cas9-edited cell line, stably expressing the receptor of interest with a fluorophore like eGFP, under its own promoter (see Note 1). In our particular example, we use a T-cell line 


\section{a Coverslips and cell preparation b TIRF Imaging}
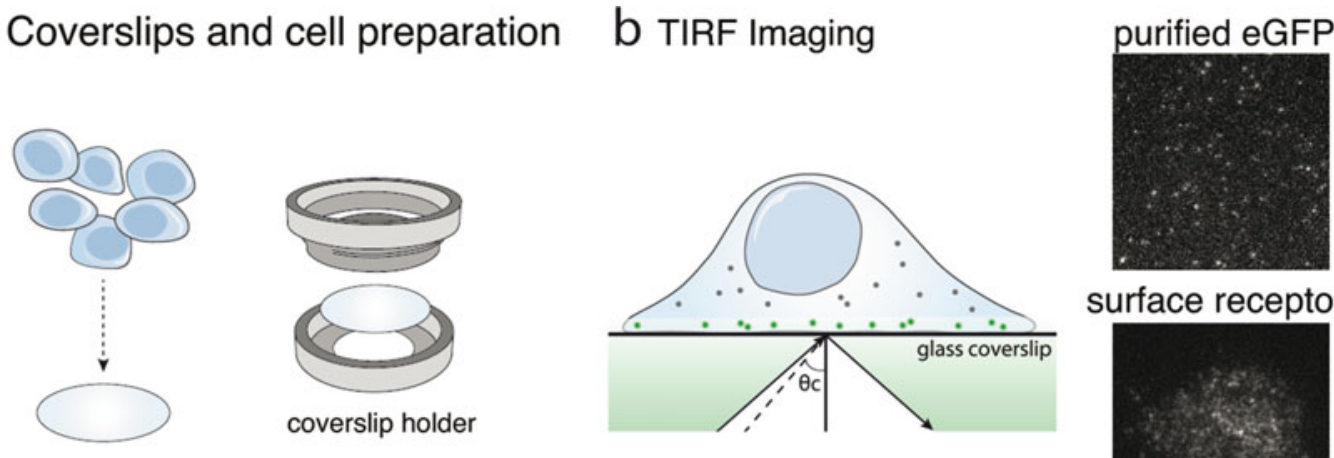

surface receptors

C Calibration for eGFP single-molecule photobleaching detection

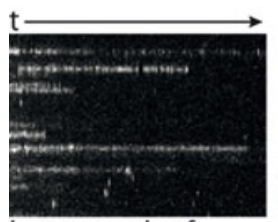

kymograph of

eGFP bleaching

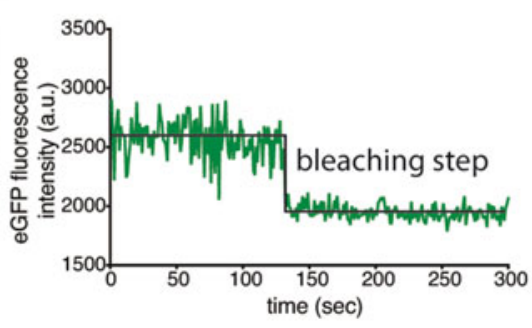

Single-molecule analysis of surface receptors

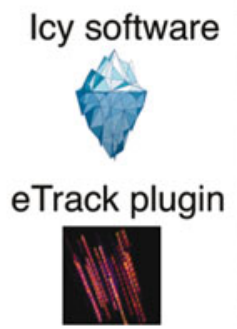

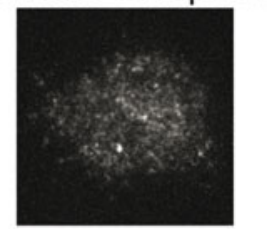

Fig. 1 Workflow for single-molecule analysis of plasma membrane receptor stoichiometry. (a) Schematic representation of cell seeding onto poly-L-lysine-coated coverslips and of the 0-ring coverslip holder used during imaging. (b) TIRF imaging allows observation of molecules close to the coverslip as purified eGFP and plasma membrane receptors. (c) Calibration for eGFP single-molecule analysis relies on photobleaching of eGFP molecules and detection of bleaching steps upon high-frequency acquisition. (d) Detection and tracking of surface receptors are done using the eTrack plug-in in Icy software

(Kit225 cells [15]) expressing the $\gamma$ chain of the IL-2R tagged with eGFP (GFP-IL-2R $\gamma$ ).

2. Roswell Park Memorial Institute (RPMI) 1640 medium containing L-glutamine and phenol red, and supplemented with $10 \%$ fetal bovine serum (heat inactivated) and $1 \mathrm{nM}$ IL-2, for cell culture at $37^{\circ} \mathrm{C}$.

3. Round $25 \mathrm{~mm}$ diameter glass coverslips No. 01 (0.13-0.17 mm thickness) (see Note 2).

4. Ethanol $70 \%$.

5. Acetone.

6. Ultrasonic cleaning bath $(220 \mathrm{~V}, 50 / 60 \mathrm{~Hz})$.

7. 6-well plate.

8. $\mathrm{ddH}_{2} \mathrm{O}$.

9. Poly-L-lysine $0.1 \%(\mathrm{w} / \mathrm{v})$. 


\section{$2.2 \quad$ TIRF Imaging}

2.2.1 Live-Image Acquisition Is Performed with an Inverted Confocal Microscope, LSM 780 Elyra PS.1 Equipped with an EMCCD Camera. The Setup Used Includes the Following Components

\subsection{Image Analysis}

1. O-ring Attofluor cell chamber (coverslip holder).

2. Purified eGFP $(1 \mathrm{mg} / \mathrm{mL})$.

3. PBS $1 \times$.

4. Cell imaging medium consisting of RPMI 1640 medium without phenol red, supplemented with $5 \%$ fetal bovine (heat inactivated).

5. Immersion oil with a refractive index of 1.518 , suited for $37^{\circ} \mathrm{C}$ imaging.

1. LSM 780 Elyra PS.1 inverted microscope, equipped with a motorized XY stage, an environmental chamber with temperature control, and a focus control by definite focus.

2. A $488 \mathrm{~nm}(100 \mathrm{~mW}) \mathrm{HR}$ - solid laser line for eGFP excitation.

3. A filter cube set with a band-pass (BP) 495-575 + low-pass (LP) 750 filter for eGFP detection.

4. An alpha Pin Apo $100 \times / 1.46$ numerical aperture (NA) oil objective.

5. An EMCCD $8871 \mathrm{~K}$ camera.

6. ZEN software.

Detection and tracking of purified eGFP and of membrane receptor are performed with Icy software (www.icy.bioimageanalysis.org) [16] and then further analyzed using Microsoft Excel.

1. Icy software version 2.0.2.0, containing the plug-ins: Spot Detector, ROI Intensity Evolution, eTrack, and Intensity Track Processor.

2. Microsoft Excel.

\section{Methods}

\subsection{Coverslips and Cell Preparation}

Glass coverslips should be washed prior to use. A washing step with ethanol and acetone, followed by an ultrasonic cleaning bath, enables removal of any oil/grease films from the surface of the glass and ensures optimal adherence of cells with minimal background. Afterwards, coverslips are coated with poly-L-lysine when working with suspension cells like T cells (Fig. la). This poly-Llysine step is unnecessary when using adherent cells.

1. Clean coverslips by immersing glass coverslips in $70 \%$ ethanol for $20 \mathrm{~min}$, then rinse in $\mathrm{ddH}_{2} \mathrm{O}$ three times, and sonicate in an ultrasonic cleaning bath for $5 \mathrm{~min}$. After, the process is repeated by immersing coverslips in acetone instead of ethanol. 
2. Coat washed coverslips by placing them in a 6-well plate with poly-L-lysine diluted in $\mathrm{ddH}_{2} \mathrm{O}$ in a ratio of $1: 10$ for $20 \mathrm{~min}$. Coverslips are then washed twice with $\mathrm{ddH}_{2} \mathrm{O}$, placed separately in a coverslip rack, and allowed to dry completely. Coated coverslips can be kept at $4{ }^{\circ} \mathrm{C}$, in a sterile sealed container, for up to 3 months.

3. Wash Kit225 GFP-IL-2R $\gamma$ once with medium without serum, and then seed them in the same medium at approximately 1.5 million cells per coverslip, in a 6 -well plate ( see Note 3 ). The plate is centrifuged at $300 \times g$ for $2 \mathrm{~min}$ to promote cell adherence. Cells can then be kept in regular RPMI medium, at $37{ }^{\circ} \mathrm{C}$ with $5 \% \mathrm{CO}_{2}$, until imaging.

\subsection{TIRF Imaging}

In order to calibrate the imaging system for the analysis of eGFP fluorescence at single-molecule level, purified eGFP should be coated onto coverslips at low density (Fig. 2a) and imaged at high acquisition frequency ( see Note 4). This calibration step is essential and needs to be performed for each experiment, using the same parameters (TIRF angle, laser power, exposure time and gain) as for the live-cell imaging. Therefore, these parameters should be first set up on a live-cell sample in order to obtain an evanescent wave and avoid, as much possible, photobleaching of the eGFP-tagged receptor (see Note 5). Importantly, acquisition of both purified eGFP and living cells should be done under a uniform field of illumination (Fig. 1b).

1. Preheat at $37^{\circ} \mathrm{C}$ the microscope chamber and coverslip holder prior imaging to avoid focus problems.

2. Prepare the purified eGFP by diluting it with PBS $1 \times$ to 1:40000 in $1 \mathrm{~mL}$ (eGFP $25 \mathrm{ng} / \mathrm{mL}$ final concentration), and add it to a glass coverslip placed on a pre-warmed coverslip holder and incubate for $30 \mathrm{~s}$ at $37^{\circ} \mathrm{C}$, in the microscope chamber. After, the eGFP-coated coverslip is washed thoroughly five times and imaged in PBS.

3. Set the TIRF acquisitions using the $100 \times / 1.46$ NA oil objective and ZEN software, with the $488 \mathrm{~nm}$ channel. The settings chosen, including exposure time, gain, laser power, and TIRF angle, must be identical to the ones used during cell imaging. As an example, the settings used were as follows: exposure time of $150 \mathrm{~ms}$, gain of 300 , laser power of $1 \%$, and TIRF angle of $64.4^{\circ}$. The eGFP sample is imaged at high frequency of $20 \mathrm{~Hz}$ ( 1 frame $/ 50 \mathrm{~ms}$ ) and recorded for a total of 300 frames.

4. Live-image the surface receptors by placing a coverslip seeded with cells on a pre-warmed coverslip holder, and carefully add $1 \mathrm{~mL}$ of imaging medium to it.

5. Image the cells at $37^{\circ} \mathrm{C}$ using the same settings as for purified eGFP imaging, with the exception of acquisition rate, which for 

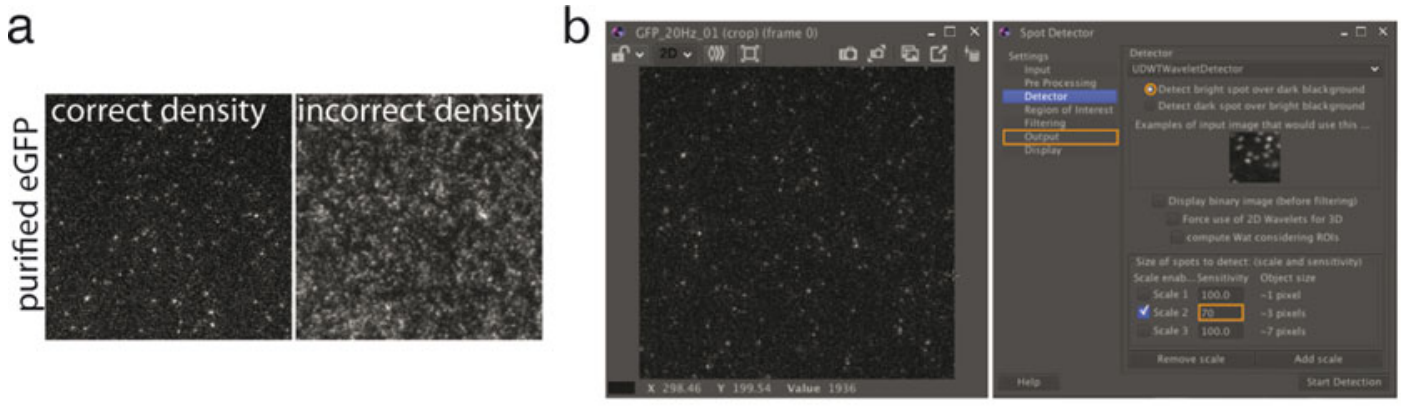

$\mathrm{C}$
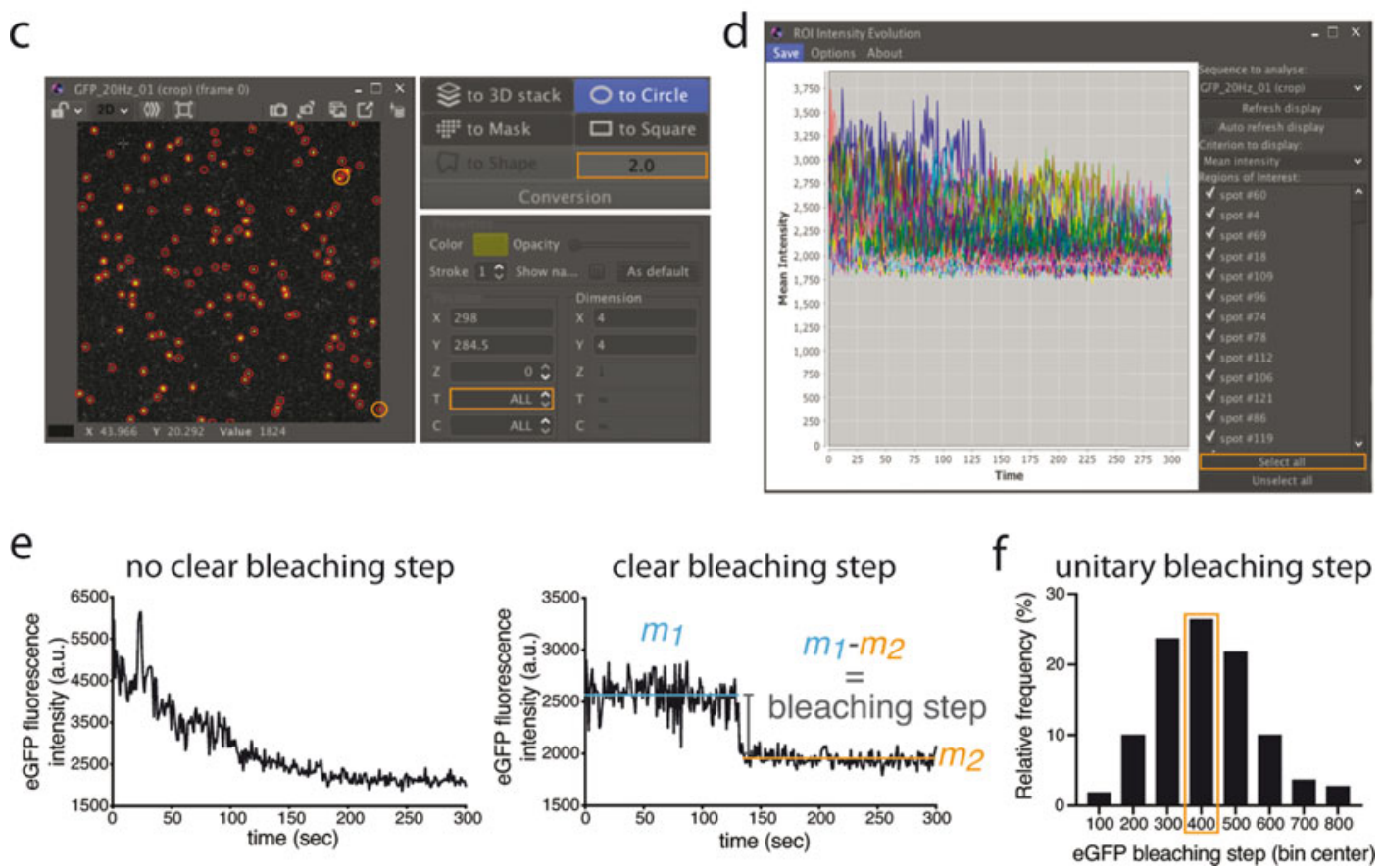

Fig. 2 Calibration for eGFP single-molecule photobleaching detection. (a) Example images of a correct density of eGFP seeded onto coverslips and an incorrect, overly dense, eGFP seeding that should be avoided. (b) Detection of eGFP spots on the first frame of the eGFP high-frequency acquisition movie using the Spot Detector plug-in in Icy. Highlighted in orange are the parameters to detect bright spots over dark background, scale and sensitivity for spot detection, and the tab Output for export of spots as ROls (step 2 of Subheading 3.3). (c) Conversion of ROls into circles with a fixed radius of two pixels from their barycenter (highlighted), copy of these ROls onto all frames of the eGFP movie (highlighted), and deletion of overlapping and border spots (highlighted) (step 3 of Subheading 3.3). (d) Detection of eGFP fluorescence intensity along time with the ROI Intensity Evolution plug-in. All spots are selected (highlighted), and their intensities are saved as an Excel file (step 4 of Subheading 3.3). (e) Example plots of eGFP fluorescence intensity along time, with an exponential decrease in intensity where no discrete bleaching step is observable (left), and one plot with a clear bleaching step (right). In plots with observable bleaching steps, the mean fluorescence intensity before $\left(m_{1}\right)$ and after $\left(m_{2}\right)$ the bleaching step is calculated, and their difference represents the photobleaching of one eGFP molecule (step 5 of Subheading 3.3). (f) The relative frequency of the unitary bleaching steps of several eGFP spots follows a Gaussian distribution. The mean of the unitary bleaching step, here with a value of $400 \mathrm{a}$. u., is later used to calculate the number of receptor molecules in endocytic tracks (step 6 of Subheading 3.3) 


\subsection{Calibration for eGFP \\ Single-Molecule Photobleaching Detection}

this assay was set to $1 \mathrm{~Hz}(1$ frame/s). Cells are imaged for a total of 150 frames, and an average of 12-15 cells are imaged per condition per experiment.

In order to calculate the number of receptor molecules present in an endocytic pit, the unitary bleaching step of the fluorophore, in this case eGFP, has to be determined using the fast acquisition movies of purified eGFP (Fig. lc).

Icy software is used to obtain the fluorescence intensity along time of the imaged eGFP. During this step, eGFP spots are automatically detected according to their approximate pixel size (scale) and brightness (sensitivity) using a wavelet-based algorithm implemented in the plug-in Spot Detector [16]. The ROIs of the detected spots are exported, and their sizes are uniformized by converting the ROIs into circles with a fixed radius from their barycenter position. Later, the intensity of GFP-IL-2R $\gamma$ spots will be acquired using the same ROI size as for eGFP (see Note 6).

By plotting the fluorescence intensity of each eGFP spot along time, it is possible to observe the bleaching step of one fluorophore and determine its intensity when discrete bleaching steps are observed (see Note 7).

1. Load the purified eGFP movie into Icy, and draw and crop a square ROI with a homogeneous illumination and a low eGFP density.

2. Detect eGFP spots by extracting the first frame of the movie, and use it as the input sequence for detection of spots with Spot Detector, within the tab Detection \& Tracking ( see Note 8). The plug-in is set to detect bright spots over dark background, in its Detector tab, and the scale and sensitivity of detection are chosen according to the spot size and brightness (see Note 9). In our case, a scale of 2 (objects of approximately 3-6 pixels size) and sensitivity of 70 are regularly used. In the Output tab of the plug-in, the Export to ROI is ticked (Fig. 2b).

3. Select and convert the exported ROIs into circles with a fixed size from their barycenter position in the section Conversion in the tab Region of Interest. A radius of 2 pixels was used for the conversion. All spots (ROIs) are then selected from the ROI panel and copied into the eGFP movie, making sure the copied ROIs are applied to all frames ( $T=A L L$ in the ROI panel). Spots that are placed on the borders of the image, and overlapping spots are suppressed (Fig. 2c).

4. Plot the intensity of the eGFP spots over time using the ROI Intensity Evolution plug-in. The eGFP movie is selected as the sequence to analyze and all ROIs are selected. Once the intensity plots of the ROIs are displayed, the results can be saved as an XLS file (Fig. 2d). 
5. Further analyze eGFP intensities using Microsoft Excel ( see Note 10). The exported data shows the mean intensity of eGFP per frame, with each column corresponding to one eGFP detected spot. Individual columns are selected, and their intensity along time is plotted. Spots that do not show clear bleaching steps are deleted (see Note 7 and Fig. 2e). Then, the mean fluorescence intensity before and after the bleaching step is calculated, and their difference corresponds to the unitary bleaching step of one molecule of eGFP (Fig. 2e).

6. Calculate the unitary bleaching steps for approximately 100 spots. If plotted, the bleaching step intensity of these spots should follow a Gaussian frequency distribution (Fig. 2f). If otherwise, it might indicate an excessive density and aggregation of eGFP. The mean of the unitary bleaching steps $\left(\mathrm{U}_{\mathrm{eGFP}}\right)$ corresponds to the intensity value of one molecule of eGFP and is later used to infer the number of GFP-IL$2 \mathrm{R} \gamma$ molecules.

3.4 Single-Molecule Analysis of Surface Receptors
When imaging receptors in living cells, the dynamics of the process have to be preserved, which prevents direct counting of molecules by photobleaching. In this case, the fluorescence intensity of the eGFP-tagged receptor can be used and divided by the unitary bleaching step of eGFP (previously determined) to infer the number of molecules in a complex.

The eTrack plug-in implemented in Icy is used for detection of surface receptors on live-imaging movies (Fig. 1d). This plug-in was developed for the detection of putative endocytic tracks (see Note 11) and uses Spot Detector to identify spots that are significantly brighter than the background, generating an elevation map of their fluorescence for the whole duration of the movie. In this elevation map, putative endocytic sites appear as high-intensity Gaussian peaks due to the multiple spot detections that are summed along the frames. In addition, eTrack relies on the $x y$ confinement of endocytic tracks over time to precisely determine the position of putative endocytic sites and successfully reconstruct them even if some detections are missing. Not all parameters of the plug-in will be mentioned here, yet the default values are usually a good starting point. The reader can refer to Bertot et al. for a description of eTrack and example movies (Movie S1 and S2) [17].

1. Load live-cell imaging movies into Icy, draw an ROI surrounding the cell (Fig. 3a), and use Spot Detector to determine the best parameters of scale and sensitivity of detection to be later introduced in the eTrack plug-in (see Note 9). These parameters may vary slightly among experiments but should be kept the same within the same experiment. 

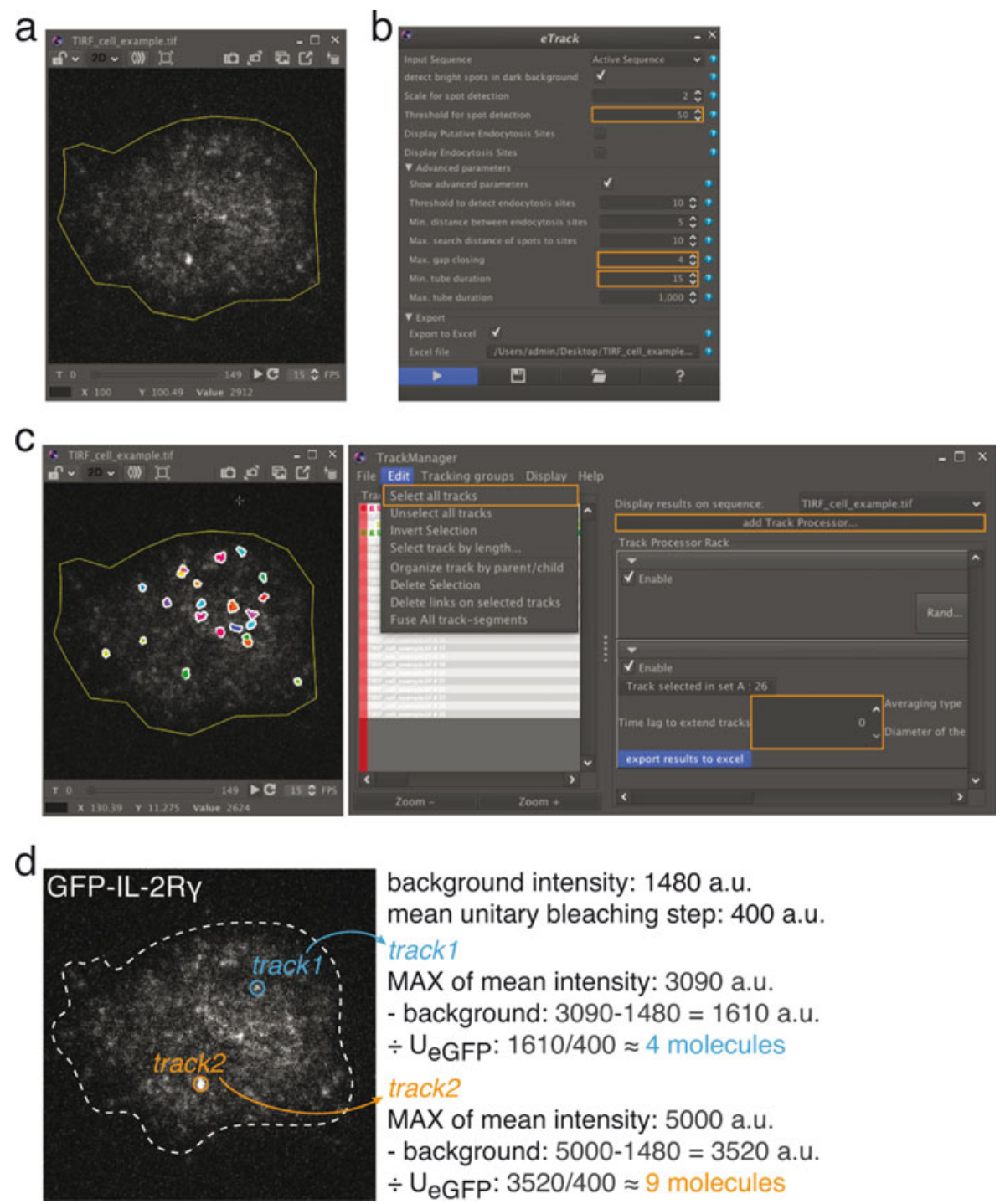

background intensity: 1480 a.u.

mean unitary bleaching step: 400 a.u.

track1

MAX of mean intensity: 3090 a.u.

- background: $3090-1480=1610$ a.u.

$\div U_{\text {eGFP: }} 1610 / 400 \approx 4$ molecules

track2

MAX of mean intensity: 5000 a.u.

- background: $5000-1480=3520$ a.u.

$\div U_{\text {eGFP: }} 3520 / 400 \approx 9$ molecules

Fig. 3 Single-molecule analysis of surface receptors. (a) Example of a TIRF movie with the ROI of one cell outlined (step 1 of Subheading 3.4). (b) eTrack panel with the parameters of threshold for spot detection, maximum gap closing, and minimum tube duration highlighted (step 2 of Subheading 3.4). (c) The resulting detected tracks will be shown on the movie (left), and their fluorescence intensity can be obtained by adding the Intensity Track Processor to the Track Manager panel (highlighted). All tracks are selected, the Time lag to extend tracks (highlighted) and the Diameter of the disk area in pixels (on the right, not shown) are defined, and results are exported to Excel (step 3 of Subheading 3.4). (d) GFP-IL-2R $\gamma$ at the cell surface with two tracks highlighted and example calculations to determine their stoichiometry based on their fluorescence intensity (MAX $I_{\text {mean }}$ ), the background fluorescence of the cell (MIN $\left.I_{\text {min }}\right)$, and the mean of the unitary bleaching steps of eGFP (MEAN $U_{\text {eGFP) }}$ (step 4 of Subheading 3.4) 
2. Analyze live-cell imaging by launching the plugin $e$ Track in the tab Detection \& Tracking. The live-cell imaging movie to be analyzed is selected as the input sequence, and the parameters previously chosen for spot detection are inserted, ensuring the plug-in is set to detect bright spots in dark background. In the advance parameters, it is possible to specify a variety of settings such as the minimum duration of the tracks (in frames) and the maximum number of missed detections (in frames) between real detections within a track (maximum gap between detections). In the case of GFP-IL- $2 \mathrm{R} \gamma$, a scale 2 and threshold of 50 are generally used for detection, and the maximum gap closing and minimum tube duration are set to 4 and 15 frames, respectively. The results are exported to Excel, by defining a file name and location, and the plug-in is started (Fig. 3b).

3. Obtain the fluorescence intensity of these tracks using the TrackManager window (automatically open), in which all detected tracks can be found. To obtain the fluorescence intensity of these tracks, first all tracks must be selected (Edit > Select all tracks) and then the Intensity Track Processor is added to TrackManager. This new processor will show as a drop-down box named Intensity profile with two parameters: the Time lag to extended tracks defines the number of frames, before and after each track, for which fluorescence intensity will be additionally recorded, and the Averaging type defines the shape of the ROI, which is usually set to DISK, with a defined Diameter of the disk area in pixels. In our case, we do not extend the fluorescence recording of the tracks (Time lag to extended tracks $=0$ ), and we detect the fluorescence intensity of the tracks in a disk ROI with four pixels of diameter, matching the purified eGFP ROIs previously obtained. Next, the tracks are added to the processor by clicking in Put current selection in trackset $A$, and results are exported to Excel (Fig. 3c).

4. Obtain the number of molecules per track by performing a post analysis of the track fluorescent intensity with the two Excel files newly created. The first one contains information on the receptor tracks (from eTrack, step 2), such as $x y$ position, duration of the track, initial and final frame, number of spots (real detections), mean spot size, and others. The second file contains data on the mean, maximum, and minimum fluorescence intensity of each track (from Track Manager, step 3 ). Importantly, the track numbers between the two files are corresponding; therefore, the fluorescence intensity of a track can be related with its duration, for example. Using the latter file, the background fluorescence of the cell is obtained by retrieving the minimum value of intensity (Excel function $M I N)$ among all frames and tracks, in the Excel worksheet Channel 0-Min intensity (see Note 12). Then, in the 
worksheet Channel 0-Mean intensity, the maximum value of intensity (Excel function $M A X$ ), for each track, is retrieved, and the background value of intensity, previously obtained, is subtracted from it. This is the fluorescence intensity value used to calculate the number of molecules in a receptor track, by simply diving it by the mean of the unitary bleaching steps of eGFP. This calculation can be described as:

$$
N_{\text {molecules }}=\frac{\text { MAX } I_{\text {mean }}-\text { MIN } I_{\text {min }}}{\text { MEAN } U_{\mathrm{eGFP}}}
$$

$N_{\text {molecules: }}$ Number of receptor molecules in a track.

MAX $I_{\text {mean }}$ : Maximum value of the mean intensity of the track.

MIN $I_{\min }$ : Minimum value of the minimum intensity of all the tracks, corresponding to the cell's fluorescence background and being a constant value used for all tracks within that cell.

MEAN $U_{\mathrm{eGFP}}$ : Mean of the unitary bleaching step values of purified eGFP (see step 6 of Subheading 3.3).

An example of this calculation for two distinct tracks is given in Fig. 3d.

\section{Notes}

1. Single-molecule photobleaching experiments rely on bright and photostable fluorophores, with eGFP being the most commonly used. Expression of receptors fused to a fluorescent protein, protein tags as SNAP- or HALO-tags, or a fluorescent ligand or antibody can be used to visualize membrane-located proteins, such as receptors. However, the presence of endogenous non-labelled receptor and the degree and stoichiometry of labeling has to be accounted for in these situations. Therefore, genome editing methods are ideal to generate cell lines in which the fluorescent tag is expressed coupled to the endogenous gene of the receptor in both alleles, thus overcoming these issues.

2. Alternatively, glass bottom MatTek plates can be used although glass coverslips allow for a better cleaning procedure.

3. Cell density should be adjusted to at least $50 \%$ confluency according to cell type. $\mathrm{T}$ cells are particularly small hence a higher cell density is required to cover the coverslip. It is crucial to wash the cells and seed them in medium without serum. The presence of serum prevents proper adhesion of cells to the coverslip, regardless of the poly-L-lysine coating.

4. Coating of eGFP at low density (lower than $1-2$ spots per $\mu \mathrm{m}^{2}$ ) is extremely important since single-molecule photobleaching counting is conditioned by the diffraction limit of light. At high 
densities of eGFP, single-molecule fluorescent spots will overlap or form aggregates, limiting the observation of single photobleaching steps. A dilution between $1: 10000$ and 1:100000 should be used for eGFP coating. The final density also varies greatly with the eGFP incubation time at $37^{\circ} \mathrm{C}$ and the number of washes. Additional washes can be done if, when starting acquisition, a slight excessive density of eGFP is seen at the microscope. Otherwise, if clustering of eGFP is noticeable, the best is to restart the preparation with an increased dilution. The reader can refer to Fig. 2a for an example of a correct and incorrect density of fluorophore coating.

5. Edited cells express endogenous amounts of the receptor coupled to one fluorophore, which usually results in a dim signal that is easily photobleached. Therefore, it is essential to choose a low laser power in order to reduce photobleaching of the tagged receptor in living cells. Additionally, low illumination intensity ensures a slow and observable stepwise photobleaching of the purified fluorophore. To increase the signal obtained, the exposure time and gain can be adjusted, taking into account that a higher exposure time will also lead to photobleaching, and a higher gain will increase background. A compromise between these parameters ensures the acquisition of suitable data for analysis. To achieve total internal reflection, the incident light has to arise from the objective at a greater angle than the critical angle for the coverslip-medium interface. In our experience, this corresponds to a TIRF angle between $62^{\circ}$ and $65^{\circ}$. For a detailed description of TIRF imaging, refer to Yildiz et al. [18].

6. The optimal radius for detection of spots should be determined so that the eGFP spots and the receptor spots fit within it yet avoiding overlapping with neighboring spots.

7. Discrete bleaching steps are seen when there is a clear abrupt decrease in fluorescence intensity of the eGFP as seen in the graph on the right in Fig. 2e. If eGFP is aggregated, multiple fluorophores will be clustered in one diffraction limited spot, and the fluorescence intensity will decrease exponentially (Fig. 2e graph on the left); hence, discrete bleaching steps will not be observed.

8. Alternatively, if the eGFP signal is dim, the initial $\approx 10$ frames can be extracted and converted into a $\mathrm{z}$-stack, and the maximum intensity projection is done. This might help with obtaining a brighter signal from the eGFP spots present during the first frames and thus a better detection with Spot Detector.

9. Different scales and sensitivities should be previously tested, according to the pixel size and brightness of the object of interest. For this optimization, the option Display binary 
image in the Detector tab of Spot Detector is of great use, allowing a clear visualization of the resulting ROIs. These parameters might vary between experiments, particularly if different conditions are being studied as, for instance, while investigating the effect of a drug on receptor endocytosis.

10. There are several "step detection" algorithms, implemented in MATLAB or other softwares, that can be used or modified to help in fast-automatic detection of single-molecule photobleaching steps, reducing the time of analysis $[19,20]$.

11. eTrack relies on the xy confinement of endocytic tracks over time, which means that tracks that are detected for the minimum number of frames set in the plug-in (minimum duration of the tracks) but that diffuse afterwards will stop being tracked and, consequently, present short durations. These tracks are not endocytic tracks since tracking was interrupted due to diffusion instead of pit invagination and exit from the TIRF plane, which occurs upon endocytosis. Therefore, the classification of tracks obtained with eTrack has to be carefully done and depends on the purpose of the study. Alternatively, other tracking methods that consider $x y$ displacement can be used.

12. The background fluorescence of the cell can also be obtained by extending the fluorescence detection of the receptor tracks, using the parameter Time lag to extended tracks in the Intensity Track Processor. This method leads to similar values of background as the determination of the minimum fluorescence intensity among all tracks. It has, however, the disadvantage that it will produce an extended fluorescence detection that might be contaminated by the fluorescence intensity of neighboring or diffusive spots that are in close proximity to the detected track. This extended fluorescence detection will always have to be considered and, preferentially, removed from further analysis.

\section{Acknowledgments}

First, we would like to thank Dr. Alexandre Grassart for training us with the technologies of CRISPR/Cas9 gene edition and singlemolecule analysis and for all helpful discussion. We thank the PBI (Imagopole) platform of Institut Pasteur for microscope maintenance and technical help. LS is part of the Pasteur Paris University (PPU) International PhD Program and has received funding from the European Union's Horizon 2020 research and innovation program under the Marie Sklodowska-Curie grant agreement No 665807. 


\section{References}

1. Clayton AHA, Walker F, Orchard SG et al (2005) Ligand-induced dimer-tetramer transition during the activation of the cell surface epidermal growth factor receptor-A multidimensional microscopy analysis. J Biol Chem 280:30392-30399

2. Liang SI, van Lengerich $B$, Eichel $K$ et al (2018) Phosphorylated EGFR dimers are not sufficient to activate Ras. Cell Rep 22:2593-2600

3. Needham SR, Roberts SK, Arkhipov A et al (2016) EGFR oligomerization organizes kinase-active dimers into competent signalling platforms. Nat Commun 7:13307

4. Huang Y, Bharill S, Karandur D et al (2016) Molecular basis for multimerization in the activation of the epidermal growth factor receptor. Elife 5:e14107

5. Nakano Y, Monde K, Terasawa H et al (2014) Preferential recognition of monomeric CCR5 expressed in cultured cells by the HIV-1 envelope glycoprotein gpl20 for the entry of R5 HIV-1. Virology 452-453:117-124

6. Colin P, Zhou Z, Staropoli I et al (2018) CCR5 structural plasticity shapes HIV-1 phenotypic properties. PLoS Pathog 14:e1007432

7. Vila-Coro AJ, Mellado M, Martín de Ana A et al (2000) HIV-1 infection through the CCR5 receptor is blocked by receptor dimerization. Proc Natl Acad Sci U S A 97:3388-3393

8. Kaksonen M, Roux A (2018) Mechanisms of clathrin-mediated endocytosis. Nat Rev Mol Cell Biol 19:313-326

9. Cocucci E, Aguet F, Boulant S et al (2012) The first five seconds in the life of a Clathrin-coated pit. Cell 150:495-507

10. Grassart A, Cheng AT, Hong SH et al (2014) Actin and dynamin 2 dynamics and interplay during clathrin-mediated endocytosis. J Cell Biol 205:721-735

11. Cocucci E, Gaudin R, Kirchhausen T (2014) Dynamin recruitment and membrane scission at the neck of a clathrin-coated pit. Mol Biol Cell 25:3595-3609

12. Lamaze C, Dujeancourt A, Baba T et al (2001) Interleukin 2 receptors and detergent-resistant membrane domains define a Clathrinindependent endocytic pathway. Mol Cell 7:661-671

13. Gesbert F, Sauvonnet N, Dautry-Varsat A (2004) Clathrin-Independent endocytosis and signalling of interleukin 2 receptors IL-2R endocytosis and signalling. Curr Top Microbiol Immunol 286:119-148

14. Basquin C, Trichet M, Vihinen $\mathrm{H}$ et al (2015) Membrane protrusion powers clathrinindependent endocytosis of interleukin-2receptor. EMBO J:1-15

15. Hori T, Uchiyama T, Tsudo M et al (1987) Establishment of an interleukin 2-dependent human $\mathrm{T}$ cell line from a patient with $\mathrm{T}$ cell chronic lymphocytic leukemia who is not infected with human $\mathrm{T}$ cell leukemia/lymphoma virus. Blood 70:1069-1072

16. de Chaumont F, Dallongeville S, Chenouard N et al (2012) Icy: an open bioimage informatics platform for extended reproducible research. Nat Methods 9:690-696

17. Bertot L, Grassart A, Lagache T et al (2018) Quantitative and statistical study of the dynamics of Clathrin-dependent and -independent endocytosis reveal a differential role of EndophilinA2. Cell Rep 22:1574-1588

18. Yildiz A, Vale RD (2015) Total internal reflection fluorescence microscopy. Cold Spring Harb Protoc 2015:pdb.top086348

19. Chen Y, Deffenbaugh NC, Anderson CT et al (2014) Molecular counting by photobleaching in protein complexes with many subunits: best practices and application to the cellulose synthesis complex. Mol Biol Cell 25:3630-3642

20. Tsekouras K, Custer TC, Jashnsaz H et al (2016) A novel method to accurately locate and count large numbers of steps by photobleaching. Mol Biol Cell 27:3601-3615 